\title{
Semantic Harmonization for Seamless Networked Supply Chain Planning in the Future of Internet
}

\author{
Ricardo Jardim-Goncalves, Joao Sarraipa, and Adolfo Steiger-Garcao \\ CTS, Uninova, Dep. ${ }^{a}$ de Eng. ${ }^{a}$ Electrotécnica, Faculdade de Ciências e Tecnologia, FCT, \\ Universidade Nova de Lisboa, 2829-516 Caparica, Portugal \\ $\{r g, j f s s, a s g\} @ u n i n o v a . p t$
}

\begin{abstract}
Today, enterprises are typically in a constant process of acquiring and updating its information technologies, however typically without an overall view of the global inter and intra enterprise's system integration. Foreseeing the future of internet, researchers have been proposing new methodologies and platforms to assist such integration in the network of applications and data. However, implementing new technologies in organizations is a difficult task, since its quality needs for architectures development are more exigent and critical than ever, due to the systems complexity and dimension, semantic needs, and to the interoperability requirements to interact with third party applications and infrastructures. Scientific foundations for EI are envisaged to roadmap such general knowledge covering the general laws of the operation on enterprise interoperability in the future of internet. This paper reports research results from ongoing European Commission supported projects that are members of the Future Internet Enterprise Systems (FInES) European Cluster ${ }^{\text {. }}$. The paper draws concepts from the complex systems science and proposes a methodology for seamless networked Supply Chain Planning (SCP), by using a domain reference ontology, data model representation standards, software components evaluation and interoperability checking processes. The methodology VALTE is used to assure that enterprises use tools for SCP compliant to semantics, represented in a common reference ontology, created by the MENTOR methodology. These two horizontal methodologies are vertically supported by interoperability checking processes, which contribute for an interoperable supply chain planning system on the future internet.
\end{abstract}

Keywords: Interoperability, Future of Internet, Semantics Interoperability, Quality Assurance, Networked Supply Chain Planning.

\section{Introduction}

The globalisation of markets and manufacturing has forced the management of supply chains not only consider business processes in the traditional value chain, but rather processes that penetrate networks of organisations. The formation of cooperation and collaboration alliances between several small organizations is proving, in multiple

\footnotetext{
${ }^{1}$ http://cordis.europa.eu/fp7/ict/enet/ei_en.html
} 
cases, to be more efficient and competitive by comparison with big companies. Thus, the research on supply chain management has turned from an intra-enterprise focus towards the one on inter-enterprise running on the internet [1].

Supply chains consist of business and technological processes, and organizational, technical, topological, informational, and financial structures. All of these processes and structures are interrelated and change with their dynamics. To ensure a high responsiveness level, the supply chain plans must be formed extremely quickly, but must also be robust [2][3]. That is why it becomes very important to plan and run supply chain plans in relation to all the structures. The increasing competitive pressures coupled with the rapid advances in information technology have brought supply chain planning into the forefront of the business practices of most manufacturing and service organizations [4]. Consequently, there has been a growing interest in electronic business (e-business) solutions to facilitate information sharing between organisations in the supply chain. However, partnerships cause some problems mainly in integrating Product Life Cycle phases, since manufacturers, distributors, designers, retailers, warehouses, often acquire their proprietary solutions which are, typically, not interoperable with another [5].

Standardisation in data structures appeared to solve the referred communication problems. Several initiatives were taken to address this issue, like ISO10303. ISO 10303, also known as STEP, is the standard for the exchange of product model data. STEP Application Protocols have been widely used in industrial environments, to support systems interoperability through the exchange of product data in manufacturing domains. However, per se data representation standards did not solve all the problems. Semantics interoperability is of major importance, and as such it is still to be solved. More recently, the development of ontologies, as promising techniques with capabilities to solve semantic issues, has been addressed by important companies and SMEs. Thus, each company is struggling to develop competencies at this ontological level, but inevitably different perspectives will lead to different final results, and achieving different ontologies in the same business domain is an actual reality. To face it, one possible solution is to have a reference ontology for a specific domain where all the domain enterprises should use in their business. Although, to force manufacturers or suppliers to adopt a specific ontology as reference is not an easy task, since each enterprise does not foresee any outcomes by changing their knowledge. Thus, an advantageous solution would be to let them to keep their terminology and classification in use, and adopt a reference ontology. The adopted ontology will be the organization knowledge front-end, enabling inter-enterprises communications sharing the same terminology and semantics. Since this reference ontology will become their front-end, each organizational enterprise should feel motivated to participate in its building process, contributing with their own terminologies, definitions and classification structure.

The paper draws concepts from the complex systems science towards the foundation for a science-base Enterprise Interoperability and proposes a methodology for seamless networked Supply Chain Planning (SCP), by using a domain reference ontology, data model representation standards, software components evaluation and interoperability checking processes. The methodology VALTE is used to assure that enterprises use tools for SCP compliant to semantics, represented in a common reference ontology, created by the MENTOR methodology. These two horizontal 
methodologies are vertically supported by interoperability checking processes, which contribute for an interoperable supply chain planning system on the future internet. Thus, the paper proposes the integration of the VALTE and MENTOR methodology, complemented by interoperability checking methods, to contribute for networked seamless supply chain planning in the future of internet. The paper finishes discussing a case study in an industrial context.

\section{Motivations for EI as a Science in the Future of Internet Domain}

As information systems in enterprises and organizations evolve and become more complex, the need for interoperable operation, automated data interchange and coordinated behavior of large scale infrastructures becomes highly critical [6]. Lack of interoperability would disturb creation of markets and will diminish innovation and competitiveness. Apart from being a technical issue, interoperability challenges also appear in the enterprises at organizational and semantic level, underlying the need for patterns and solutions that support the seamless cooperation among ICT systems, information and knowledge, organizational structures and people [7].

Enterprise Interoperability (EI) is recognized as a high-impact productivity factor both within the private and the public sector, affecting the overall quality, yield time and cost of transactions, design and manufacturing operations or digital public services [8]. Up to now, the principal tools for targeting the above challenges appear as the various standards that try to govern information systems development and operation [9]. Such standards are usually linked with specific market sectors, application areas or technology trends, thus having a limited time span, a static nature and quite often different interpretations by technology vendors and users[9][11].

However, in spite of the research developed so far, nowadays it was not established yet the scientific foundations for EI. This is a deficit recognized by the EI research community, disabling the generalization and complete reuse of the methods and tools that have been developed [12][13].

\subsection{Open Research Questions on EI}

EI suggests that organizations can seamlessly interoperate with others throughout research development of focal areas, removing barriers to interoperability, fostering a new networked business culture, and transferring and applying the research results in industrial sectors. These areas are within the scientific domains of systems complexity, network science, information theory and web science. With them, the scientific foundations related with the major EI research topics can be worked out, connected with the results of the applied research that has been developed by the EI research community [13]. Among the most relevant research results achieved for EI, we identify of special interest for the establishment of the scientific foundations those on distributed systems, shared data and knowledge, evolutive applications, dynamics and adaptation of networked organizations on a global scale. Those are all directly related with rapid evolution of technology and applications, plug and play instruments, self monitoring capabilities, benchmarking and evaluation of degrading processing, automatic or on demand reprocessing, recompiling or fixing of components or processes. Moreover, to achieve a steady stable EI in a global scale 
there is the need for human assisted supervising systems supported by embedded supervising systems with learning capabilities.

Nevertheless, the role of standardisation policy is a major global and regional tool in EI context (e.g., ISO, CEN). Standards must point out to be perfect, completely clear but they must be implemented by the market. To reach globalization objectives for EI, they must be submitted to robust feedback mechanisms aimed to receive input from implementers, interested communities and from the market in order to assure a dynamic improving and standards maintenance. Scientific methods to assess the suitability, impact and the extension of the adoption and relevance of such standards, i.e., based on statistical methods, in the EI domain must be tightly adopted. Thus, the science foundation for EI must be well specified and general, completely unambiguous, designed to be flexible, robust and predicable in the global context, refraining from dependencies on technology and usage.

\subsection{EI as a Science for Future of Internet}

There are some European research projects researching in this field (e.g., iSurf, KNET, COIN, CoSpaces, ATHENA, INTEROP) [6], but all are concentrated in developing focal solutions for specific business scenarios, in an applied research perspective, and yet none is conducting the researching towards such generalization in a scientific foundation ground [13]. One of the aims is to establish the scientific and technological ground to allow different "systems node" to be integrated in a collaborative network, advancing at its own needs, keeping interoperable in the network where it wants to be integrated. Thus, it will permit advanced adaptation and optimization of systems, e.g., supporting their maintenance processes by the use of technologies suitable for generalized knowledge representation applied to the Model Management (MoM) domain, namely dynamic models-morfisms (DynamicMoMo).

With the foreseen research results, the adoption of advanced techniques for metamodeling and automatisms for model and data transformations, will enable to have the engine for interoperability not embedded directly in the systems coding, but through proper adaptative techniques get a suitable characterization of the actual status of the system's morphisms, supporting predictive system evolution, and analysis of its complexity in the dynamics of the network, including the respective transients and systems responsive behavior. At knowledge level, it is foreseen the need for the harmonization of ontological structures within and between the different network nodes, supported by statistical methods (e.g., stochastic methods) to permit semantic adaptability for the users specificities and to support the application dynamics. Then, enrichment of the semantic mapping will be possible, as a process to gather, classify, describe and then analyse the semantically features in the domain of the system models, and take better decisions in the advent of uncertainty [14][15].

The following research topics have been recognized as part of the core for the establishment of EI as a science:

1- Intelligent reconfiguration of components, for interoperability maintenance of evolutive networked systems.- Learning and adaptability: After indentified the need to solve an interoperability problem, the related systems typically know very few about the necessities required to have the global system completely interoperable. A learning process should be designed to support the adaption of the several system 
network nodes involved, and thus keep the global network interoperable.- Transient analysis: The global interoperable network, as a complex integrated system, will face transients whenever an internal or external "interference" occurs, e.g., update in one of its nodes. Thus, there will be a period of time which the systems nodes need to react and readapt to before the system becomes again stable and interoperable. The evolution and progressive adaptation of each network system node should be done supported by a systematic study and analysis of the network transients, as single node, clusters, and global network.- Interoperability checking: The global network needs to be checked and assessed to assure the maintenance of the networked interoperable system. A proper methodology for monitoring, diagnosis and prognosis, should be in place to assure the interoperability of the complex system in the advent of dynamics in the network.

2- Conformance testing and Interoperability checking for complex systems interoperability assessment- Discovery and Notification: When a new system node is integrated in the network, or it is updated, how such updates can be automatically identified and completely recognized by the network, and how the network should react to become interoperable, or keep its interoperability, with the new node, or update, through the automatic understanding of the intrinsic knowledge and behaviour of the node. Then, what such information can be processed and what are the needed adaptations of the systems node, to have the global network again globally interoperable.- Automatized catagorization of ontological structures: Automatized development of ontologies from descriptive specifications in non specialized language, e.g., queries described in natural language, supported by an engine with feedback for the user, with learning and reconfiguration capacities.- Conformance checking: The evolution of the network, by the integration of a new node or updates in the existing ones, will required checking for the conformance of data, models, knowledge and behaviours of the systems and applications. A proper methodology should be in place to assure such conformity in the advent of such dynamics.

3 - Harmonization of ontological structures to support the application dynamics and enable adaptability of users semantical specifications- Mutation of ontologies supported by stochastic methods: Mutation of ontologies using stochastic method to support the updates in the representation of concepts and its instances.Harmonization of ontologies and semantical adaptability: Semantic harmonization, and adaptative mapping in dynamic environments, with mediation of semantic conflicts according to the interactions and evolution with the systems which it interacts.- Adaptative services for knowledge management: Knowledge is the basis for seamless interoperability of the integrated global network. Adaptative services for knowledge management will assure the accuracy of the information and behaviour of the complex system in each node and in the integrated network, support the dynamics and evolutionary characteristics of the complex system.

\section{Networked Enterprise Reference Ontology for Interoperability}

The development of an enterprise reference ontology can follow the MENTOR methodology [16]. Its main objective is to help an organization to adopt or use and to build, a domain reference ontology, after through several main steps as semantic 
comparisons, basic lexicon establishment, mappings among ontologies and others operations on knowledge base representations. The method to support the development of a common reference ontology for a group of enterprises sharing a business domain, provides several steps as semantic comparisons, basic lexicon establishment, mappings among ontologies and other operations on ontologies. This method is composed by two phases with three steps each (Fig. 1): the Lexicon Settlement - Phase 1 (steps: 1; 2 and 3), and the Reference Ontology Building - Phase 2 (steps: $4 ; 5$ and 6 ). All of these steps are deeply described in the following two pictures where each step has a set of actions which has a number related to the step which belongs to (e.g. 1.1 is an action of the step 1).

The Lexicon Settlement phase (steps: 1; 2 and 3) represents a domain knowledge acquisition which comparatively to the human language apprentice phase could be represented in computer science as a semantic organized structure with definitions.

The thesaurus can represent such words structure of associated meanings and thus should be built in order to establish the lexicon of a specific domain. This phase has three steps: Terminology Gathering (step 1); Glossary Building (step 2) and Thesaurus Building (step 3). These steps define a set of workflows that establishes a thesaurus of the domain before starting the ontology building.

Figure 1 (left part) depicts the state diagram of the lexicon settlement phase. The terminology gathering step concerns to the process of collecting all relevant terms (action 1.2) in a specific domain previously defined (action 1.1). All the participants in the process should give their inputs. There is no rule from where the terms should come. Since they are related with the domain established. Tools for automatic extraction of domain related terms can be found, nevertheless there is always need of a human checking before close the terms list to not miss any domain terms. All the terms provided from the contributors are acceptable in this step (action 1.2). Nobody has authority to erase other's participant term. The term should be collected with reference to the contributor in order each contributor provide term's annotation in the next step (action 2.1).

Glossary is a specialized vocabulary with corresponding annotations. This vocabulary includes terms that are unique to the subject, have special meaning in the field of interest. The annotations include descriptive comments and explanatory notes for the terms, such as definitions, synonyms, and references. A Glossary can be used when communicating information in order to unify knowledge sharing. The Glossary Building step (step 2) intends to build a glossary in the domain defined. It starts with annotations attribution (action 2.1) to the terms collected. Each contributor should provide the annotations for his own terms. After having all the terms provided with annotations, it proceeds to the terms revision cycle (actions: 2.2; 2.3 and 2.4). In this cycle it could be useful to use a multi-language dictionary (action 2.0) in case of the organization members don't use the same natural language. The dictionary will help translations to the agreed language for the reference ontology. The terms revision process can have semantic and syntactic cases of mismatches (action 2.3), where they are recorded as a semantic mismatch for future mappings using the proposed mediator ontology. 

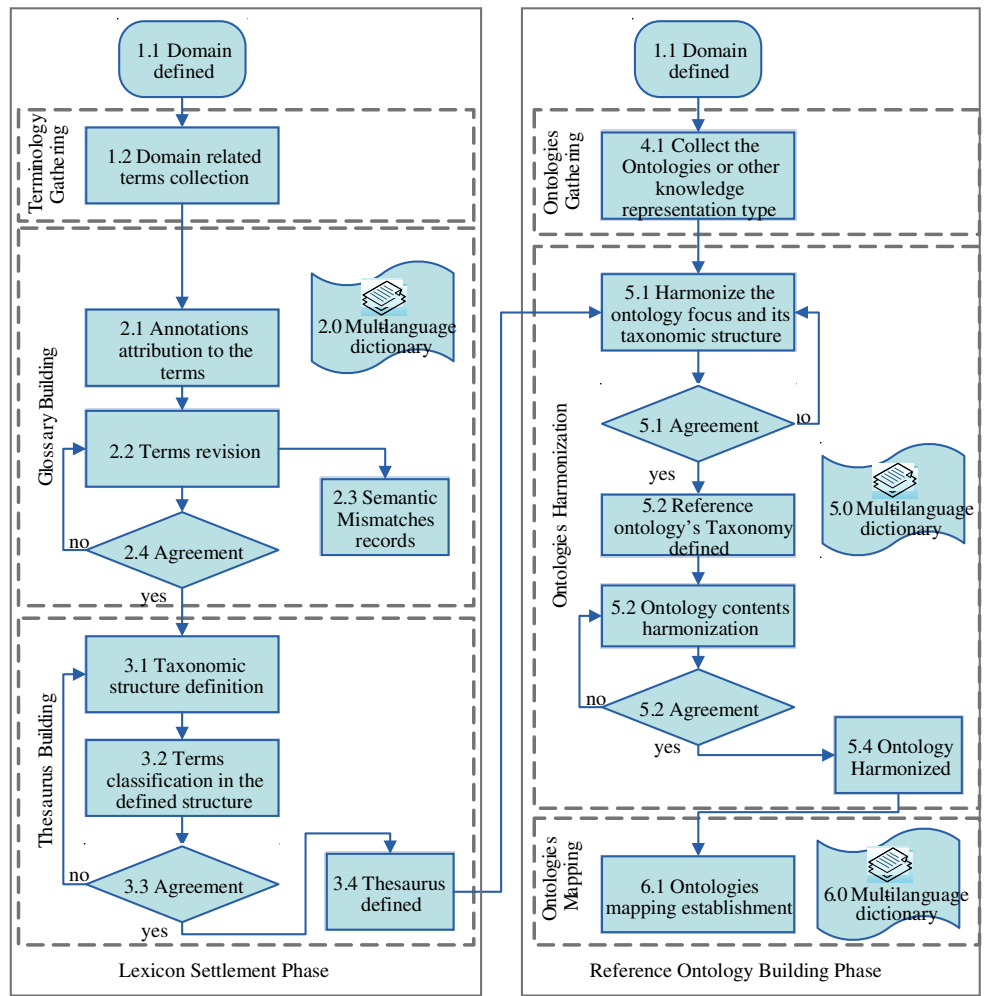

Fig. 1. MENTOR Phases and steps

After a careful revision in all the terms (action 2.2) with a successful agreement (action 2.4) in their meaning consolidation, the glossary is defined from the terminology list in the domain specified. Another output from this process is the semantic mismatch records (action 2.3): this is made using the Mediator Ontology. The Thesaurus Building step (step 3) is composed by a cycle where firstly, the knowledge engineers define a taxonomic structure (action 3.1) from the glossary terms, establishing some as thesaurus node terms. Secondly, the other terms are classified to the right paths in the existent taxonomic structure, being the thesaurus leafs (action 3.2). If there is an agreement (action 3.3) in the structure and in the terms classified, the thesaurus is defined (action 3.4). If not, the cycle starts again from the taxonomic structure definition (action 3.1). The thesaurus defined will enhance the ontology harmonization process in the next phase. The Reference Ontology Building phase - Phase 2 (steps: 4; 5 and 6) is the phase where the reference ontology is built and the semantic mappings between the organizational ontologies and the reference one is established. Figure 1 (right part) describes this.

The first step comprehends ontologies gathering (action 4.1) in the previously domain defined (action 1.1). Other type of knowledge representation could be used as input for the harmonization ontologies process together with the thesaurus defined (action 3.4) in the previous phase. The harmonization method for building ontologies, 
proposes the development of a single harmonized Ontology's by two cycles (actions: 5.1 and 5.3) where first the structure is discussed until having agreement on it (action 5.1), which result on the definition of the common classes and the class hierarchy (action 5.2), and then the same process for the ontology contents definition (action 5.3). From this process new semantic conflicts could be found. After agreement, the resolution could be recorded in the Mediator Ontology for further mapping establishments. With all the agreements accomplished, the harmonized ontology is finalized (action 5.4) together with the mapping tables (action 6.1), describing the ontological relationships between the harmonized ontology and each one of the individual ontologies through the use of the semantic mismatches records (action 2.3).

Semantic difficulties related to the natural language of the potential users of the harmonized ontology are likely to happen. To assist on it, the ontology is complemented with a multi-language dictionary where a set of normalized tokens gives the reference to the corresponding concepts and definitions in different native languages (actions 5.0 and 6.0).

\subsection{VALTE: Evaluation Methodology for Supply Chain Software Components}

The essential parts of software quality evaluation are the quality model, the method of evaluation, software measurement, and the supporting tools [17]. To develop good software, quality requirements should be specified, the software quality assurance process should be planned, implemented and controlled, and both intermediate products and end products should be evaluated [18]. VALTE is an evaluation methodology for supply chain software components, using as reference the Software Product Quality Evaluation Reference Model that describes the process, activities and tasks performed during the quality evaluation of a software product [19]. This reference models is defined by the standard [20] that contains general requirements for specification and evaluation of software quality and clarifies the general concepts providing a process description for evaluating quality of software product, stating the requirements for the application of the evaluation process. This specification is part of the SQuaRE series of standards created by ISO (the International Organization for Standardization) and IEC (the International Electrotechnical Commission). SQuaRE replaces the current [21] series and the [22].

\section{Case Study}

This case study reports on a real case analysed during European Commission supported research projects. To guarantee the survival in today's competitive and demanding digital world of business, the European companies, especially SMEs, should be more agile, self-sustainable and responsive to the changes in the supply chain. Obtaining and maintaining a competitive edge in supply chain is not only the concern of individual SMEs, but should be also addressed by the entire chain jointly. The supply chain partners should collaborate effectively so as to better align supply and demand forecasts to have a joint strategy for handling exceptions that will occur in the way of realizing the "the network is the business" vision, nowadays on top of the internet. The simple choice of furniture components suppliers by a furniture 
manufacturer brings interoperability problems. Suppliers have defined various nomenclatures for their products and its associated knowledge. Thus, the need to align applications, to exchange products data and semantics emerged as a priority to solve the dilemma. Figure 2 describes the validating scenario, where a set of enterprises agreed to work together to supply a big common client with various furniture products which are built collaboratively.

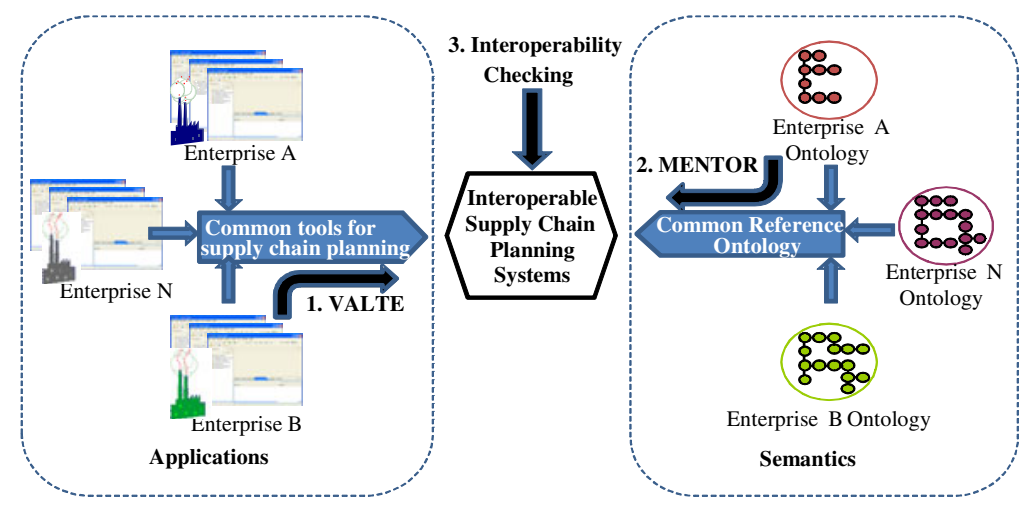

Fig. 2. Case Study overview [23]

The first step is to follow the VALTE methodology (left part of the Fig. 2), which will guide the applications evaluation activities. The evaluation follows a plan that includes the list of evaluation modules to be applied against the defined requirements related to the desired level of the software components characteristics. The evaluation results are then stated based on the metrics and measures defined and the positive or negative response of the software components evaluation to all the tests performed defines if the applications belongs to the set of common tools for a specific supply chain planning.

In the second step it is developed a reference ontology to the enterprises that are working together in this supply chain to establish between them a common semantics (right part of the Fig. 2). The MENTOR methodology is used to develop such reference ontology. During the reference ontology building phase, it is produced a mediator ontology which records all the semantic operations performed in this process. One of the applications of these semantic operations logs is to use that recorded information for semantic translation. One possible example of such process is when a message with a product request is sent to Enterprise B. The mediator ontology is used to get the "semantic translation" of the information present in the message, which uses syntax accordingly to the reference ontology, to the equivalent syntax used in the Enterprise B.

To ensure the interoperability between the systems, the third step on this use case, it is applied the Conformance Testing (CT) to its exchanged files. Based on the defined methodology for CT, the architecture shown in the Figure 3, is used to validate such files. The architecture was designed based in web-services, able to receive the files in XML format and checking them against the reference testing model using an Application Engine developed in JAVA, SAX, Schematron and XALAN. 


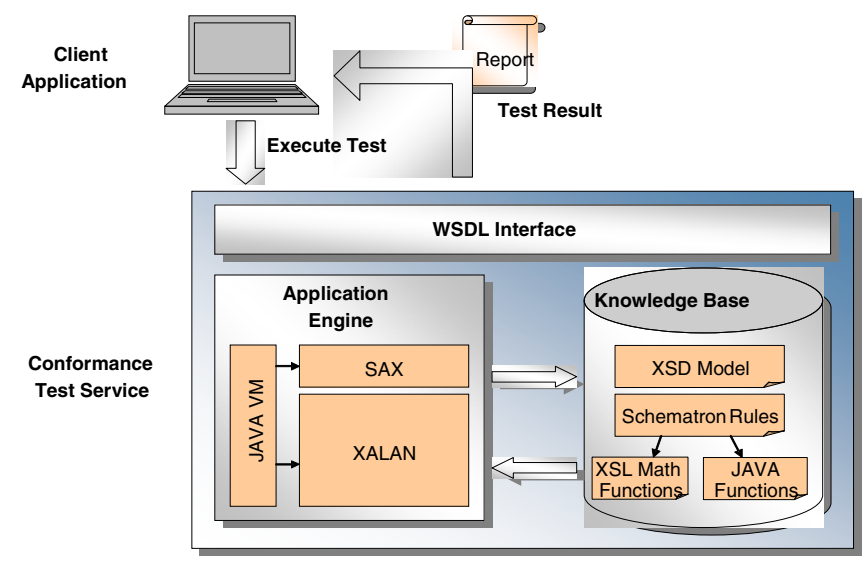

Fig. 3. Architecture for CT system validation [24]

Using the CT the user can check the files against the defined models, ensuring its correct implementation. The CT checks the XML against syntactic and semantic rules and sends back the detected errors enabling its correction. With CT executed to its XML files, the next step is the application of the Interoperability Checking (IC). To apply IC, the user will analyze and modify the test files, sent by the IC system, and send it back to evaluation. After check all the files, defined in the Abstract Test Suites (ATS) for IC, the user receives the confirmation that its system is interoperable. With all the ATS executed (CT ATS and IC ATS), the system validation can ensure that the systems are in conformance with the model defined and is interoperable with others system of this type.

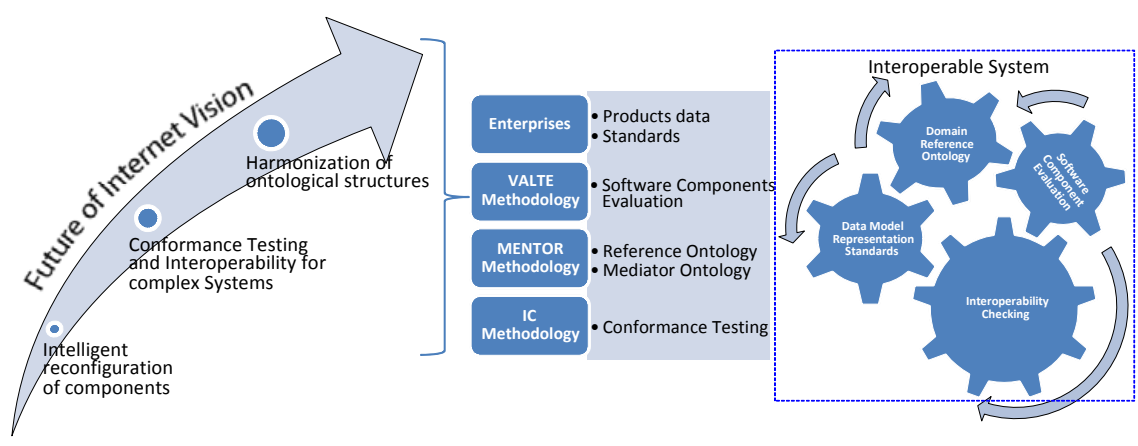

Fig. 4. System interoperability methodologies in Future of Internet Vision

\section{Conclusions}

System methodologies for networked enterprise interoperability facilitate organizations to keep its technical and operational environment, improving its methods of work and the usability of the installed technology through quality 
assurance of the system software components, ontological harmonization of the enterprises product models in use, assessed by a fitting validation framework for conformance testing and interoperability checking. However, to have an enterprise organizational system interoperable, it has to have a domain reference ontology which enhances inter-enterprise's semantics interoperability concerning to the contents of a standardized data representation model. These both components (reference ontology; data representation model) should be complemented with software quality assessment and Interoperability checking methodology able to perform model conformance testing (Fig. 4).

The proposed methodology enable the computational systems of any set of enterprises which work together in a networked supply chain planning to smoothly communicate between each other using syntax and semantic present in data representation standards and in the reference ontology respectively. This is complemented with a previous software components evaluation and a post conformance testing procedures.

Such methodology was applied with good results in a real scenario supervised by the research EuropaINNOVA initiative through the INNOVAfun and iSurf projects (member of the European Future Internet Enterprise Systems (FInES) Cluster). These achievements have been encouraging to the development of further framework functionalities in the future, like the generation of the reports according to a normative schema (e.g., defined in EXPRESS and XML), to enable automatic inference and reasoning on the errors found, and provide automatic correction of the identified errors by an internet-based expert system.

Acknowledgments. This work has been partly funded by the European Commission (E.C.) through EuropaInnova and IST projects: INNOVAfun - 031139; and iSURF IST 213031. The authors acknowledge the E.C. and all the project partners for their support during the development of various ideas and concepts presented in the paper.

\section{References}

[1] Rudberg, M., Klingenberg, N., Kronhamn, K.: Collaborative supply chain planning using electronic marketplaces. Integrated Manufacturing Systems Journal 13(8), 596-610 (2002)

[2] Jardim-Goncalves, R., Grilo, A.: SOA4BIM: Putting the building and construction industry in the Single European Information Space, Automation in Construction (2009)

[3] Grilo, A., Jardim-Goncalves, R.: Value proposition of interoperability on BIM and collaborative working environments. J. Automation in Construction AUTOCON (2009)

[4] Gupta, A., Maranas, C.D.: Managing demand uncertainty in supply chain planning. Computers and Chemical Engineering 27, 1219-1227 (2003)

[5] Jardim-Goncalves, R., Grilo, A., Steiger-Garcao, A.: Developing interoperability in mass customization information systems. Mass Customization Information Systems in Business. Idea (January 2007), ISBN-10: 1599040395

[6] (follow link PUBLIC DELIVERABLES) Major worldwide repository with publications from research in the area of Interoperability, http: / /www. interop-vlab.eu/

[7] Jardim-Goncalves, R., Grilo, A., Steiger-Garcao, A.: Challenging the Interoperability in the Construction Industry with MDA and SoA. Computers in Industry 57(8-9), 679-689 (2006) 
[8] Farinha, F., Steiger-Garcao, A., Jardim-Goncalves, R.: Integration of cooperative production and distributed design in AEC. Advances in Engineering Software 38(11-12), 772-779 (2007)

[9] Steiger-Garcao, A., Jardim-Goncalves, R., Farinha, F.: An open platform for interoperability of civil engineering enterprises. In: Advances in Engineering Software (2007)

[10] Jardim-Goncalves, R., Steiger-Garcao, A.: Implicit Multilevel Modelling in Flexible Business Environments. Comm. of the ACM 45(10) (2002), ISSN 0001-0782

[11] Figay, N., Steiger-Garcao, A., Jardim-Goncalves, R.: Enabling interoperability of STEP Application Protocols at meta-data and knowledge level. International Journal of Technology Management 36(4) (2006), ISSN 0267-5730

[12] Leyton, M.: A Generative Theory of Shape. LNCS. Springer, Heidelberg, ISBN-10: 3540427171

[13] Enterprise Interoperability Research Roadmap, European Commission, ftp: / / ftp.cordis.europa.eu/pub/ist/docs/ict-ent-net/ ei-research-roadmap-v5final_en.pdf

[14] Jardim-Goncalves, R., Sarraipa, J., Agostinho, C., Panetto, H.: Knowledge Framework for Intelligent Manufacturing Systems. Int. J. of Intelligent Manufacturing (2009)

[15] Sarraipa, J., Jardim-Goncalves, R.: MENTOR: An Enabler for Interoperable Intelligent Systems. International Journal of General Systems (2009)

[16] Sarraipa, J., Silva, J., Jardim-Goncalves, R., Monteiro, A.: MENTOR - A Methodology for Enterprise Reference Ontology Development. In: 2008 4th International IEEE Conference on Intelligent Systems (2008)

[17] Suryn, W., Abran, A.: ISO/IEC SQuaRE. The 2nd generation of standard for quality of software product. In: Proceedings of 7th IASTED International Conference on Software Engineering and Applications, SEA 2003, Marina del Rey, CA, USA, November 3-5 (2003)

[18] Trudel, S., Lavoie, J.-M., Paré, M.-C., Suryn, W.: The design of the software quality evaluation method combining CMMI and ISO/IEC 14598: the context of a small company. In: Proceedings of 12th International Software Quality Management \& INSPIRE Conference (BSI), Canterbury, Kent, UK, April 5-7 (2004); Article a obtenu le prix du meilleur article scientifique 2004

[19] Vieira, H., Jardim-Goncalves, R.: VALTE - Methodology for VALidation and TEsting of Supply Chain Software Components. In: 15th International Conference on Concurrent Enterprising (2009)

[20] ISO/IEC CD 25040: Software engineering - Software product Quality Requirements and Evaluation (SQuaRE) - Evaluation reference model and guide. International Organization for Standardization, Switzerland

[21] ISO/IEC 9126-1: Software Engineering-Software product quality-Part 1: Quality model. Geneva. International Organization for Standardization, Switzerland

[22] ISO/IEC 14598-1: Software product evaluation-Part 1: General overview. Geneva. International Organization for Standardization, Switzerland

[23] Sarraipa, J., Vieira, H., Agostinho, C., Jardim-Goncalves, R.: Methodology for Seamless Supply Chain Planning. In: The International Conference on Software, Services \& Semantic Technologies Varna, Bulgaria (2009)

[24] Wiles, A.: Relevance of Conformance Testing for Interoperability Testing. ACATS ATSCONF (2003) 